\title{
Evaluation of urea and creatinine levels in vaginal wash fluid for the diagnosis of premature rupture of membranes
}

\author{
Abha Sharma, Richa Sharma*, Tannavi Agarwal \\ Department of Obstetrics and Gynecology, UCMS and GTB Hospital, Delhi, India \\ Received: 21 May 2020 \\ Accepted: 30 June 2020 \\ *Correspondence: \\ Dr. Richa Sharma, \\ E-mail: gautamdrricha1@gmail.com \\ Copyright: (c) the author(s), publisher and licensee Medip Academy. This is an open-access article distributed under \\ the terms of the Creative Commons Attribution Non-Commercial License, which permits unrestricted non-commercial \\ use, distribution, and reproduction in any medium, provided the original work is properly cited.
}

\begin{abstract}
Background: Evaluation of urea and creatinine levels in vaginal wash fluid for the diagnosis of premature rupture of membranes.

Methods: The study was conducted on150 pregnant patients, 50 in each group. Confirmed PROM and unconfirmed PROM. Per speculum examination was done to look for pooling, $\mathrm{pH}$ tested using the Pehanon paper and vaginal wash fluid was collected. Vaginal wash fluid urea and creatinine levels were tested by a kit based on spectrophotometry.

Results: The mean urea levels were $26.35 \mathrm{mg} / \mathrm{dl}$ in the study Group 1 and $3.12 \mathrm{mg} / \mathrm{dl}$ in the control group. ROC curve was plotted and the cut off value of vaginal wash fluid urea was found to be $8.55 \mathrm{mg} / \mathrm{dl}$. The vaginal wash fluid urea levels of $>8.55 \mathrm{mg} / \mathrm{dl}$ detected PROM with a sensitivity, specificity, negative and positive predictive value of $100 \%$. The mean creatinine levels were $0.62 \mathrm{mg} / \mathrm{dl}$ in study Group 1 and $0.20 \mathrm{mg} / \mathrm{dl}$ in the control group. ROC curve was plotted and the cut off value of vaginal wash fluid creatinine was found to be $0.405 \mathrm{mg} / \mathrm{dl}$. Vaginal wash fluid creatinine levels detected PROM with a sensitivity of $76 \%$ and specificity of $100 \%$. The negative predictive value and positive predictive values were $80.4 \%$ and $100 \%$.

Conclusions: Urea can be used as a definite marker of PROM and creatinine can be used as a supportive marker.
\end{abstract}

Keywords: Creatinine levels, Definite marker, Premature rupture of membranes, Urea level

\section{INTRODUCTION}

Premature rupture of membranes (PROM) is defined as rupture of fetal membranes occurring prior to the onset of labour. The incidence of PROM has remained constant through the years and has been reported to be between 3 $18.5 \%$. Preterm premature rupture of membranes (PPROM) refers to the occurrence of this event prior to 37 weeks gestation and accounts for about one fourth of all cases of ruptured membranes. ${ }^{1}$ Numerous risk factors are associated with PROM. Patients at higher risk include smokers, lower socioeconomic status, history of sexually transmitted infections, previous preterm delivery, vaginal bleeding, polyhydramnios, multifetal pregnancy and after procedures like cerclage, amniocentesis. ${ }^{2}$
A patient with symptoms suggestive of PROM should have a prompt evaluation. The evaluation includes patient's history and per-speculum examination done to evaluate gross pooling of the amniotic fluid or leaking from cervix. If PROM cannot be confirmed by PS examination, authors can use the nitrazine paper test, the fern test or have a sonographic evaluation of amniotic fluid volume. The diagnosis can also be achieved by identifying the presence of specific amniotic fluid markers in vaginal environment. These include measurement of prolactin, $\alpha$-fetoprotein, di-amine oxidase, insulin like growth factor binding protein-1 (IGFBP-1), human chorionic gonadotropin, fetal fibronectin and placental alpha microglobulin-1 (amnisure). ${ }^{3}$ 
All these tests have advantages and drawbacks. The ideal test would be simple, rapid, inexpensive and non-invasive with good sensitivity and specificity. Up to now there is no gold standard non-invasive test for diagnosis of PROM3. The only definitive test is the USG guided dye instillation test using indigo carmine or Evans blue dye.

Correct diagnosis of PROM has great importance because failure of diagnosis can lead to unwanted obstetric complications like chorioamnionitis, endometritis, cord compression, cord prolapse and fetal distress, fetal malpresentation, placental abruption, risk of operative delivery and neonatal sepsis.

On the other hand, overdiagnosis can lead to unnecessary interventions like induction of labour and hospitalization with its cost implications. ${ }^{4}$

The management of patients with PROM remains controversial. The major prognosticating variables at time of membrane rupture are gestational age and residual amount of amniotic fluid. ${ }^{5}$ Patients with PROM <32 weeks gestation should be managed conservatively, whereas those at or beyond 34 weeks can be delivered. The management of patients between 32-34 weeks is unclear. Some authors recommend collection of amniotic fluid for fetal lung maturity testing and expectant management until 34 weeks unless fetal lung maturity test is positive. Therefore, a correct diagnosis of PROM becomes imperative to formulate management policy in these patients. ${ }^{5}$

The combination of patient's history, per speculum examination, nitrazine test and the fern test for evaluation of patients with symptoms suggestive of PROM yields a sensitivity of only $93 \% .^{6}$ Amnisure which has the best sensitivity and specificity is quite expensive, as are other biochemical markers of PROM; moreover their availability may be a problem at several centers.

It has been proposed that vaginal fluid urea and creatinine may be helpful in diagnosing PROM because fetal urine is the most important source of amniotic fluid in the second half of pregnancy. Creatinine concentration in amniotic fluid increases gradually between 20 and 32 weeks of gestation and more rapidly thereafter, when they are two to four times higher than maternal serum.

Very few studies have been done to evaluate vaginal fluid urea and creatinine as a marker of PROM. Kafali et al, showed sensitivity, specificity, positive predictive and negative predictive value of $100 \%$ in detecting PROM by this method. ${ }^{7}$

The current study is being undertaken to evaluate vaginal fluid urea and creatinine concentration to detect PROM in symptomatic patients. If it shows a good diagnostic accuracy, then this will be a much cheaper as well as easily available alternative to the current tests and costwise a big help in developing country like ours.
The aim of the study was to evaluate urea and creatinine levels in vaginal wash fluid as a marker of premature rupture of membranes.

\section{METHODS}

A cross sectional study was conducted in the Labour Room and outpatient of department of obstetrics and gynecology and department of biochemistry, of study institution.

A total of 150 pregnant women with $\geq 28$ weeks of pregnancy were recruited. They were divided into study group and control group as follows:

\section{Study group}

The study group comprised of 100 patients admitted in labour room with the history of leaking per vaginum and was subdivided into Group 1 and 2:

Group 1: Consisted of 50 pregnant women with the history of leaking per vaginum with both amniotic fluid pooling in the vagina on per speculum examination and positive $\mathrm{pH}$ paper test (confirmed PROM).

Group 2: Consisted of 50 pregnant women with the history of leaking per vaginum with either both amniotic fluid pooling in the vagina and $\mathrm{pH}$ paper test negative or only one of these positive (unconfirmed PROM).

\section{Control group}

Consisted of 50 pregnant women well matched for age and gestational age without any complaints and were recruited from outpatient department.

An informed written consent for the study was obtained from all patients after explaining the nature of the study.

At admission a detailed history was taken as per proforma including the time since leaking.

\section{Inclusion criteria}

- All patients were subjected to complete general and physical examination including obstetrical examination.

\section{Exclusion criteria}

- Congenital malformations of fetal urinary system

- Bleeding per vaginum

- Patients in labour

- Presence of meconium in liquor.

Their per-speculum examination was done and presence or absence of pooling in the posterior fornix was noted. Vaginal wash fluid collection was done during per speculum examination. $5 \mathrm{ml}$ of sterile saline was instilled 
into the posterior fornix and $3 \mathrm{ml}$ of it was aspirated out with the same syringe and sent to the laboratory for centrifugation and estimation of urea and creatinine.

The $\mathrm{pH}$ of the pooled fluid was tested using the pehanon paper. This paper is a green strip, which has a $\mathrm{pH}$ range of 5-7.5.

The colour changes from green to blue with increasing $\mathrm{pH}$. Change to blue colour was taken as a positive test.

In patients with history of leaking per vaginum if both pooling and $\mathrm{pH}$ test were positive patients were put in confirmed leaking group (study Group 1). If only one test was positive or both tests were negative patient was put in unconfirmed leaking group (study Group 2). Patients without history of leaking and with both tests negative were put in control group.

\section{Method of urea and creatinine estimation}

Vaginal wash fluid urea and creatinine levels were tested by a kit based on spectrophotometry. Urea estimation was done by DAM method and estimation of creatinine was done by Jaffe's Alkaline Picrate method.

\section{Management}

Patients were managed as per the protocol of the institution and were followed-up till delivery.

\section{Data analysis}

\section{Outcome parameters}

- Vaginal wash fluid urea and creatinine levels.

\section{Comparison between groups}

All the groups were compared with regards to demographic profile and vaginal wash fluid urea and creatinine levels.

Sensitivity, specificity, positive predictive value and negative predictive value of vaginal wash fluid urea and creatinine levels were calculated for the diagnosis of premature rupture of membranes.

\section{Statistical analysis}

Comparison between groups was be done by one-way ANOVA test followed by Tukey's test. Receiver operating characteristic curve analysis was used to establish an optimal cut off value of vaginal fluid urea and creatinine. The normality was tested for urea and creatinine level and found both variables were not normally distributed.

Non-parametric Krusal Wallis test was applied to compare among the three groups.

Pair-wise comparison was done using Mann-Whitney U test and p-value was adjusted using Bonferroni corrections that is $0.05 / 3=0.017=0.02$. A p-value of $<0.001$ was taken as significant.

\section{RESULTS}

Confirmed PROM (study Group 1) consisted of 50 patients with both pooling and positive $\mathrm{pH}$ test. In unconfirmed PROM (study Group 2) $46 \%$ patients had only pooling positive, $40 \%$ had only $\mathrm{pH}$ test positive and $14 \%$ had both negative (Table 1).

Table 1: Group allocation.

\begin{tabular}{|c|c|c|c|c|}
\hline & \multirow{2}{*}{$\begin{array}{l}\text { History of } \\
\text { leaking }\end{array}$} & \multicolumn{3}{|c|}{ Examination findings } \\
\hline & & Pooling & Positive pH test & $\begin{array}{l}\text { Both pooling and } \mathrm{pH} \\
\text { test negative }\end{array}$ \\
\hline Study Group 1 ( $\mathrm{n}=50)$ (confirmed leaking) & 50 & 50 & 50 & 0 \\
\hline Study Group 2 ( $\mathrm{n}=50)$ (unconfirmed leaking) & 50 & 23 & 20 & 7 \\
\hline Control Group $(n=50)$ (no leaking) & 0 & 0 & 0 & 50 \\
\hline
\end{tabular}

Table 2: General characteristics of all the three groups.

\begin{tabular}{|llllll|}
\hline & & $\begin{array}{l}\text { Study Group 1 (n=50) } \\
\text { (confirmed leaking) }\end{array}$ & $\begin{array}{l}\text { Study Group 2 (n=50) } \\
\text { (unconfirmed leaking) }\end{array}$ & $\begin{array}{l}\text { Control Group } \\
\text { (n=50) (no leaking) }\end{array}$ & $\begin{array}{l}\text { p- } \\
\text { value }\end{array}$ \\
\hline Mean age (years) & & $23.44 \pm 2.589$ & $23.40 \pm 3.037$ & $23.30 \pm 3.190$ & 0.971 \\
\hline \multirow{3}{*}{ Parity } & P0 & 29 & 24 & 26 & \\
& P1 & 18 & 22 & 20 & \\
\cline { 2 - 5 } & P2 & 3 & 4 & 4 & 3.729 \\
\hline \multicolumn{2}{l}{ Mean period of gestation (weeks) } & $34.86 \pm 1.885$ & $34.98 \pm 1.970$ & $35.11 \pm 1.916$ & 0.804 \\
\hline
\end{tabular}


Table 3: Amniotic fluid index in study Group 1 and 2.

\begin{tabular}{|llll|}
\hline AFI* & $\begin{array}{l}\text { Study Group 1 (confirmed } \\
\text { leaking) }(\mathbf{n = 5 0})\end{array}$ & $\begin{array}{l}\text { Study Group 2 (unconfirmed } \\
\text { leaking) }(\mathbf{n = 5 0})\end{array}$ & p-value \\
\hline$<5$ & $4(8 \%)$ & $1(2 \%)$ & 0.362 \\
\hline$\geq 5$ & $46(92 \%)$ & $49(98 \%)$ & \\
\hline Mean AFI & $8.63 \pm 1.858$ & $8.928 \pm 1.652$ & \\
\hline
\end{tabular}

*Oligohydramnios is diagnosed when amniotic fluid index $<5$.

Table 4: Vaginal wash fluid urea levels in study Group 1 and control Group.

\begin{tabular}{|c|c|c|c|}
\hline $\begin{array}{l}\text { Vaginal wash fluid } \\
\text { urea level (mg/dl) }\end{array}$ & $\begin{array}{l}\text { Study Group } 1 \text { (confirmed } \\
\text { leaking) }(n=50)\end{array}$ & $\begin{array}{l}\text { Control Group (no leaking) } \\
(\mathbf{n = 5 0})\end{array}$ & p-value $*$ \\
\hline$<5$ & 0 & 48 & 0.00 \\
\hline $5.1-10$ & 0 & 2 & \\
\hline $10.1-15$ & 1 & 0 & \\
\hline $15.1-20$ & 4 & 0 & \\
\hline $20.1-25$ & 24 & 0 & \\
\hline $25.1-30$ & 21 & 0 & \\
\hline Mean urea (mg/dl) & 26.35 & 3.12 & \\
\hline
\end{tabular}

Table 5: Distribution of patients according to cut off value of urea in vaginal wash fluid in all the groups.

\begin{tabular}{|llll|}
\hline $\begin{array}{l}\text { Vaginal wash fluid } \\
\text { urea level (mg/dl) }\end{array}$ & $\begin{array}{l}\text { Study Group 1 (confirmed } \\
\text { leaking) }(\mathbf{n = 5 0 )}\end{array}$ & $\begin{array}{l}\text { Study Group 2 (unconfirmed } \\
\text { leaking) }(\mathbf{n}=\mathbf{5 0})\end{array}$ & $\begin{array}{l}\text { Control Group (no } \\
\text { leaking) }(\mathbf{n}=\mathbf{5 0})\end{array}$ \\
\hline$<8.55$ & $0(0 \%)$ & $7(14 \%)$ & $50(100 \%)$ \\
\hline$\geq 8.55$ & $50(100 \%)$ & $43(86 \%)$ & $0(0 \%)$ \\
\hline
\end{tabular}

Table 6: Effectiveness of vaginal wash fluid urea level.

\begin{tabular}{|c|c|}
\hline \multicolumn{2}{|l|}{ Urea level } \\
\hline Sensitivity & $100 \%$ \\
\hline Specificity & $100 \%$ \\
\hline Negative predictive value & $100 \%$ \\
\hline Positive predictive value & $100 \%$ \\
\hline
\end{tabular}

The groups were well matched for age, parity and period of gestation with a p-value of $>0.05$ as shown in the Table 2. If presence of oligohydramnios is taken as criteria for leaking then only $8 \%$ of patients had oligohydramnios in the confirmed leaking group and $2 \%$ in the unconfirmed group. This was not statistically significant (p-value >0.001) (Table 3).

The vaginal wash fluid urea levels were significantly higher in women with confirmed leaking as compared to women without leaking ( $\mathrm{p}$-value 0.00 , Table 4). As per ROC curve the cut off value of vaginal fluid urea was found to be $8.55 \mathrm{mg} / \mathrm{dl}$.

All patients with confirmed leaking had vaginal wash fluid urea levels higher than the cut off value whereas all control group patients had vaginal wash fluid urea levels less than the cut off value.

Table 7: Vaginal wash fluid creatinine levels in study Group 1 and control Group.

\begin{tabular}{|llll|}
\hline $\begin{array}{l}\text { Vaginal wash fluid creatinine } \\
\text { level }(\mathbf{m g} / \mathbf{d l})\end{array}$ & $\begin{array}{l}\text { Study Group 1 (confirmed leaking) } \\
(\mathbf{n = 5 0 )}\end{array}$ & $\begin{array}{l}\text { Control Group (no leaking) } \\
(\mathbf{n = 5 0 )}\end{array}$ & \begin{tabular}{l} 
p-value* \\
\hline $0.10-0.30$
\end{tabular} \\
\hline $0.31-0.50$ & 12 & 40 & 0.00 \\
\hline $0.51-0.70$ & 7 & 10 & \\
\hline $0.71-0.90$ & 13 & 0 \\
\hline $0.91-1.1$ & 3 & 0 \\
\hline$>1.1$ & 3 & 0 \\
\hline Mean creatinine $(\mathrm{mg} / \mathrm{dl})$ & 0.62 & 0 \\
\hline
\end{tabular}

*ANOVA test. 
Table 8: Distribution of patients according to cut off value of creatinine in vaginal wash fluid in all the groups.

\begin{tabular}{|llll|}
\hline $\begin{array}{l}\text { Vaginal wash fluid } \\
\text { creatinine level }(\mathbf{m g} / \mathbf{d l})\end{array}$ & $\begin{array}{l}\text { Study Group 1 (confirmed } \\
\text { leaking) }(\mathbf{n = 5 0 )}\end{array}$ & $\begin{array}{l}\text { Study Group 2 (unconfirmed } \\
\text { leaking) }(\mathbf{n = 5 0})\end{array}$ & $\begin{array}{l}\text { Control Group (no } \\
\text { leaking) }(\mathbf{n = 5 0})\end{array}$ \\
\hline$<0.405$ & $12(24 \%)$ & $18(36 \%)$ & $50(100 \%)$ \\
\hline$\geq 0.405$ & $38(76 \%)$ & $32(64 \%)$ & $0(0 \%)$ \\
\hline
\end{tabular}

In study Group 2 (unconfirmed leaking) $86 \%$ patients with either pooling or $\mathrm{pH}$ test positive had urea levels higher than the cut off value while rest $14 \%$ which had both pooling and $\mathrm{pH}$ test negative had urea levels lower than the cut off (Table 5).

The vaginal wash fluid urea levels of $>8.55 \mathrm{mg} / \mathrm{dl}$ detected PROM with a sensitivity and specificity of $100 \%$. The negative and positive predictive value for the presence of PROM were also 100\% (Table 6).

The vaginal wash fluid creatinine levels were significantly higher in women with confirmed leaking as compared with control group without any leaking (pvalue 0.00) (Table 7).

As per ROC curve the cut off value of creatinine was found to be $0.405 \mathrm{mg} / \mathrm{dl}$.

In patients with confirmed leaking $76 \%$ had vaginal wash fluid creatinine levels higher than the cut off value whereas all control group patients had vaginal wash fluid creatinine levels less than the cut off value.

In study Group 2 (unconfirmed leaking) 64\% patients with either pooling or $\mathrm{pH}$ test positive had creatinine levels higher than the cut off value while rest $36 \%$ which had creatinine levels lower than the cut off value included 7 patients with both pooling and $\mathrm{pH}$ test negative Table 8 .

Table 9: Effectiveness of vaginal wash fluid creatinine level.

\begin{tabular}{|ll|}
\hline Creatinine level \\
\hline Sensitivity & $76 \%$ \\
\hline Specificity & $100 \%$ \\
\hline Negative predictive value & $80.4 \%$ \\
\hline Positive predictive value & $100 \%$ \\
\hline
\end{tabular}

The vaginal wash fluid creatinine levels detected PROM with a sensitivity of $76 \%$ and specificity of $100 \%$. The negative predictive value and positive predictive values were $80.4 \%$ and $100 \%$ respectively (Table 9 ).

Vaginal wash fluid urea levels were significantly higher in all patients irrespective of gestational age. Vaginal wash fluid creatinine levels were higher than cut off value in $20 \%$ patients in $\leq 34$ weeks and $56 \%$ patients in $>34$ weeks. The difference was not statistically significant (p-value 0.178).

There is no significant correlation either linear or nonlinear between urea and creatinine levels and period of gestation (Table 10).

Table 10: Vaginal wash fluid urea and creatinine according to period of gestation in group 1 (confirmed leaking).

\begin{tabular}{|llll|}
\hline Cut off values & $\mathbf{3 4}$ weeks & $>\mathbf{3 4}$ weeks & p-value \\
\hline Urea $<8.55$ & $0(0 \%)$ & $0(0 \%)$ & Not possible \\
\hline Urea $\geq 8.55$ & $14(28 \%)$ & $36(72 \%)$ & \\
\hline Creatinine $<0.405$ & $4(8 \%)$ & $8(16 \%)$ & 0.178 \\
\hline Creatinine $\geq 0.405$ & $10(20 \%)$ & $28(56 \%)$ & \\
\hline
\end{tabular}

A total $74 \%$ patients presented within 8 hours of leaking and $8 \%$ patients presented after 12 hours of leaking. There is no significant correlation either linear or nonlinear between urea and creatinine levels and duration of leaking (Table 11).

Most of the patients delivered within 7 days of PROM (86\% and $80 \%)$.

A total $22 \%$ patients in Group 1 and $10 \%$ in Group 2 had delivery by caesarean section.
Table 11: Duration of leaking in study group 1 and its correlation with vaginal wash fluid urea and creatinine levels.

\begin{tabular}{|ll|}
\hline $\begin{array}{l}\text { Duration of } \\
\text { leaking (hours) }\end{array}$ & $\begin{array}{l}\text { No. of patients in study group } 1 \\
\text { (confirmed leaking) }(\mathbf{n}=\mathbf{5 0})\end{array}$ \\
\hline$\leq 4$ & $20(40 \%)$ \\
\hline $4.1-8$ & $17(34 \%)$ \\
\hline $8.1-12$ & $9(18 \%)$ \\
\hline$>12$ & $4(8 \%)$ \\
\hline
\end{tabular}


Table 12: Outcome of cases in study group.

\begin{tabular}{|llll|}
\hline Variables & $\begin{array}{l}\text { Study group 1 (confirmed } \\
\text { leaking) }(\mathbf{n = 5 0 )}\end{array}$ & $\begin{array}{l}\text { Study group 2 (unconfirmed } \\
\text { leaking) }(\mathbf{n = 5 0})\end{array}$ & p-value \\
\hline PROM delivery interval (days) & & $40(80 \%)$ & \\
\hline$\leq 7$ & $43(86 \%)$ & $10(20 \%)$ & 0.522 \\
\hline$>7$ & $7(14 \%)$ & $7.07 \pm 10.04$ & 0.102 \\
\hline Mean \pm SD & $4.32 \pm 5.88$ & & \\
\hline Mode of delivery & & $45(90 \%)$ & \\
\hline NVD & $39(78 \%)$ & $5(10 \%)$ & 0.134 \\
\hline LSCS & $11(22 \%)$ & $15(30 \%)$ & \\
\hline NICU admission & $18(36 \%)$ & & \\
\hline Apgar score (5min) & & $7(14 \%)$ & \\
\hline$<7$ & $13(26 \%)$ & $43(86 \%)$ & \\
\hline$\geq 7$ & $37(74 \%)$ & & \\
\hline
\end{tabular}

A total $36 \%$ neonates required NICU facilities in Group 1 and $30 \%$ in Group 2 immediately after delivery (Table 12).

\section{DISCUSSION}

Premature rupture of membranes occurs in approximately $3-18 \%$ of patients, and results in the loss of the natural protection of the fetus and intra uterine contents from bacterial invasion. Consequently, both mother and fetus are at a greater risk of infection. Preterm PROM is a complication of $2 \%$ to $20 \%$ of all pregnancies and is an important contributor of perinatal morbidity and mortality. $^{2}$ The major cause of perinatal morbidity and mortality associated with PPROM is prematurity. Morbidities related to prematurity include respiratory distress syndrome, necrotizing enterocolitis, interventricular hemorrhage, cerebral palsy, and sepsis. Maternal risks of term PROM include chorioamnionitis and postpartum endometritis. ${ }^{3}$

There has been controversy among health care professionals about the optimal approach for diagnosis and management of prematurely ruptured membranes. In most cases, membrane rupture can be confirmed by documenting amniotic fluid leakage from the os or with visualization of pooling in the posterior fornix. In approximately $20 \%$ to $25 \%$ of cases, rupture of membranes is not grossly apparent. A patient's history may suggest membrane rupture, but test results are nonconfirmatory, creating an obstetrical dilemma. Early and accurate diagnosis of PROM would help in proper management and minimize the complications4. More importantly, if patient is preterm, the decision to either continue or terminate the pregnancy depends upon the accurate diagnosis of PROM.

Several tests have been described to diagnose rupture of membranes other than history and per speculum examination. These include fern test, $\mathrm{pH}$ test and use of amniosense pad. Biochemical markers include estimation of fetal fibronectin, di amine oxidase, prolactin, glucose, insulin like growth factor binding protein, urea and creatinine estimation. As yet there is no confirmatory non-invasive diagnostic test for PROM. The ideal test would be simple, noninvasive, rapid and cheap. ${ }^{4}$

A new marker for PROM was suggested by Kafali et al, i.e. estimation of urea and creatinine in vaginal wash fluid. ${ }^{7}$ Very few studies are available in literature using urea and creatinine levels in vaginal wash fluid for diagnosing PROM. The present study was undertaken to evaluate the vaginal wash fluid urea and creatinine as a marker for diagnosis of PROM.

\section{General characteristics}

Age

In the present study the mean ages were well matched in all the 3 groups (p value 0.971 ). It was $23.44,23.40$ and 23.30 in the study Group 1, study Group 2 and control group respectively. Though age is not considered a significant risk factor associated with PROM. Most of the patients in the present study were below 25 years of age. This could be due to early age of child bearing in the population of northern India.

\section{Parity}

In the present study most of the patients were primiparous and were well matched in all the 3 groups ( $p$ value 0.729 ). Though parity is not a proven risk factor for PROM, Ladfor's et al suggested increased risk of PROM in primiparous patients.

\section{Gestational age}

The gestational age at which PROM occurs is important in determining the management and outcome of patients. Preterm PROM complicates $2-20 \%$ of all deliveries, in the present study the mean gestational age in weeks were 
34.86, 34.98, 35.11 in study Group 1, study Group 2 and control group respectively and were well matched ( $p$ value 0.804$)$. The mean period of gestation in a similar study of vaginal wash fluid urea, creatinine estimation by Kafali et al were 35.6, 38.2 and 40.1 weeks in the confirmed leaking group, unconfirmed leaking group and control group respectively. ${ }^{7}$ While in the study by Gurbuz et al it was 36.67 and 35.29 in the PROM and control group. 8

\section{Ultra sound assessment of AFI}

In the present study ultrasound assessment of AFI was done to determine whether it helped in the diagnosis of rupture of membranes. An AFI of $<5$ was taken as oligohydramnios thus indicating leaking. Only $8 \%$ patients in confirmed leaking group showed oligohydramnios. The mean AFI in the present study was 8.63 and $8.92 \mathrm{~cm}$ in the confirmed and unconfirmed leaking group, which was not significant statistically ( $p$ value 0.362 ).

This was similar to the study by Robson et al that showed no difference in mean depth of amniotic fluid in patients with confirmed PROM, compared with those in whom PROM was not confirmed, the frequency of oligohydramnios was $5 \%$ and $5.8 \%$ respectively. ${ }^{9}$ Their results show that ultrasound estimation of amniotic fluid is of no value in the diagnosis of PROM.

\section{Urea levels in vaginal wash fluid}

The vaginal wash fluid urea levels were significantly higher in women with confirmed leaking (study Group1) as compared to women without leaking (control Group) ( $p$ value 0.00 ). In the confirmed leaking group $98 \%$ of patients had urea levels between $15-30 \mathrm{mg} / \mathrm{dl}$ while $96 \%$ patients in the control group had urea levels below 5 $\mathrm{mg} / \mathrm{dl}$. The mean urea levels were $26.35 \mathrm{mg} / \mathrm{dl}$ in the study Group1 and $3.12 \mathrm{mg} / \mathrm{dl}$ in the control group.

This was similar to the study by Kafali et al in which the mean vaginal fluid urea level was $34.6 \mathrm{mg} / \mathrm{dl}$ in the confirmed PROM group and $1.3 \mathrm{mg} / \mathrm{dl}$ in the control group, where the difference was statistically significant $(\mathrm{p}<0.001){ }^{4,7}$

The cut off value of urea was calculated using ROC curve, it was found to be $8.55 \mathrm{mg} / \mathrm{dl}$ for the present study. In the study by Kafali et al the cut off was $12 \mathrm{mg} / \mathrm{dl}$.

\section{Predictive accuracy of urea levels in vaginal wash fluid}

All patients of confirmed leaking had vaginal wash fluid urea levels higher than cut off value whereas all control group patients had urea levels less than cut off value.

Amongst the unconfirmed leaking group $86 \%$ patients had urea levels higher than the cut off value. $14 \%$ patients had urea levels lower than the cut off value and these were the same patients in which pooling and $\mathrm{pH}$ were both negative.

Taking the cut off value of $8.55 \mathrm{mg} / \mathrm{dl}$, the vaginal wash fluid urea estimated PROM with a sensitivity, specificity, negative and positive predictive value of $100 \%$. Considering the sensitivity and specificity of urea levels as $100 \%, 14 \%$ patients in the unconfirmed leaking group had a false positive history of leaking. Thus, history of leaking per vaginum in diagnosis of PROM comes out to have a sensitivity of $100 \%$ but a positive predictive value of $86 \%$ only.

\section{Creatinine levels in vaginal wash fluid}

The vaginal wash fluid creatinine levels were significantly higher in women with confirmed leaking (study Group 1) as compared to women without leaking (control Group) (p value 0.00). In the present study, the mean creatinine levels were $0.62 \mathrm{mg} / \mathrm{dl}$ in study group1 and $0.20 \mathrm{mg} / \mathrm{dl}$ in the control group. This was similar to the study by Gurbuz et al in which the mean vaginal wash fluid creatinine levels were 0.70 and $0.026 \mathrm{mg} / \mathrm{dl}$ in the case and control Group, while in the study by Kafali et al the mean vaginal wash fluid creatinine levels were 1.5 and $0.28 \mathrm{mg} / \mathrm{dl}$ respectively in confirmed and control group. ${ }^{7,8}$

The cut off value of vaginal wash fluid creatinine for the diagnosis of PROM was calculated using the ROC curve, it was found to be $0.405 \mathrm{mg} / \mathrm{dl}$ for the present study.

The vaginal wash fluid mean creatinine values for control group are almost same in all the 4 studies. The values for the confirmed leaking group differs from $0.6 \mathrm{mg} / \mathrm{dl}$ in the present study to $1.5 \mathrm{mg} / \mathrm{dl}$ in Kafali's study. The difference could be because of the difference in the mean gestational age of the study patients as creatinine is known to increase with increasing gestational age. ${ }^{8}$

The different values for cut off (from $0.12-0.9 \mathrm{mg} / \mathrm{dl}$ ) in different studies could also be explained by this.

\section{Predictive accuracy of creatinine levels in vaginal wash fluid}

In the present study $76 \%$ patients in study Group 1 and $64 \%$ patients in study Group 2 had creatinine levels higher than the cut off whereas all control group patients had a vaginal wash fluid creatinine levels less than the cut off value. $36 \%$ patients in unconfirmed leaking group had creatinine levels lower than the cut off value, these included $14 \%$ patients in which all 3 tests (pooling, $\mathrm{pH}$ test, and urea estimation) were negative indicating absence of leaking.

Taking the cut off value of creatinine as $0.405 \mathrm{mg} / \mathrm{dl}$ the vaginal wash fluid creatinine estimated PROM with a sensitivity of $76 \%$, negative predictive value of $80.4 \%$ and specificity and positive predictive value of $100 \%$. 


\section{Correlation of vaginal wash fluid urea and creatinine levels with the period of gestation}

The amniotic fluid urea and creatinine levels are known to gradually increase with increasing gestational age. As the urea and creatinine levels were measured in vaginal wash fluid in confirmed leaking group comprising of patients from 28-38 weeks of gestation, the present study sought to find a correlation between these levels and gestational age as it could provide an additional benefit of indicating the maturity of the fetus.

Oliviera et al found that the mean urea and creatinine levels were $29.41 \mathrm{mg} / \mathrm{dl}$ and $1.28 \mathrm{mg} / \mathrm{dl}$ at $27-34$ weeks gestation, $31.52 \mathrm{mg} / \mathrm{dl}$ and $1.83 \mathrm{mg} / \mathrm{dl}$ at $36-42$ weeks gestation. $^{10}$ Creatinine concentration of $2 \mathrm{mg} / \mathrm{dl}$ represented an age of at least 37 weeks. In the study by Deshpande et al they suggested that a creatinine concentration of $1.75 \mathrm{mg} / \mathrm{dl}$ or more correlated significantly with a gestational age of 37 weeks or more and the amniotic fluid creatinine levels can be used as a marker of fetal maturity. ${ }^{11}$

In the present study vaginal wash fluid urea levels were significantly higher in all patients irrespective of gestational age while creatinine levels were higher than cut off value in $20 \%$ patients in $\leq 34$ weeks and $56 \%$ patients in $>34$ weeks but the difference was not statistically significant.

To see whether urea and creatinine estimation in vaginal wash fluid increased over the period of gestation in the present study, the vaginal wash fluid urea and creatinine levels were plotted against the period of gestation in the confirmed leaking (study Group 1) patients. No significant correlation either linear or nonlinear was found between urea and creatinine levels and period of gestation. This could be because of small sample size $(n=50)$ in the present study and also only 2 of the patients were of less than 32 weeks gestational age. Further studies with larger cohorts of patients at various gestational ages are required to ascertain whether vaginal wash fluid creatinine levels in patients of PROM could be of additional benefit in ascertaining the maturity of fetus.

\section{Correlation of vaginal wash fluid urea and creatinine levels with the duration of leaking}

Literature shows that in several tests for diagnosing PROM, the false positive and false negative rates increased with the increase in duration since leaking. ${ }^{1}$ To see whether urea and creatinine estimation in vaginal wash fluid also differed over increasing duration of leaking in the present study, the urea and creatinine levels were plotted against the duration of leaking in the confirmed leaking (study Group 1) patients.

In the present study $40 \%$ patients presented within 4 hours, $74 \%$ within 8 hours and $8 \%$ after 12 hours of leaking.
No significant correlation either linear or nonlinear was found between urea and creatinine levels and duration of leaking. The inference being that the efficacy of diagnosing PROM by urea and creatinine estimation in vaginal wash fluid does not change significantly with increasing duration of leaking.

\section{Outcome measures}

\section{PROM delivery interval}

As literature suggests, there exists an inverse relationship between gestational age and PROM delivery interval and also oligohydramnios is associated with a shorter latency period. ${ }^{4}$ In PROM patients $50 \%$ go into labour in 1 day and $70 \%$ within 3 days while in PPROM almost $90 \%$ patients deliver within 2 weeks. $^{2}$

In the present study $86 \%$ patients in the confirmed leaking (study Group 1) and $80 \%$ patients in unconfirmed leaking group delivered within 1 week. $20 \%$ patients in unconfirmed leaking had PROM delivery interval $>1$ week, these included those 7 patients who presented with history of leaking but all the 4 tests i.e., $\mathrm{pH}$, pooling and urea, creatinine were negative. These patients did not have leaking and were kept on conservative management thus having a longer latency period.

The PROM delivery interval in patients in the study by Kafali et al were 1.2 and 5.5 weeks in the confirmed and unconfirmed group with a $\mathrm{p}$ value of $<0.0017$. While in the present study it was 4.32 and 7.07 days in the confirmed leaking (study Group 1) and unconfirmed leaking (study Group 2) ( $\mathrm{p}$ value 0.522). The greater PROM delivery interval in the present study could be explained by the fact that in the present study the mean gestational ages were 34.8 and 34.9 weeks while in Kafali's study it was 35.6 and 38.2 weeks in the confirmed and unconfirmed groups. The lesser mean gestation in the present study resulted in an increase in the latency and a longer duration of conservative management. The difference in the PROM delivery interval between study Group 1 and study Group 2 was not statistically significant because $86 \%$ patients in Group 2 also had leaking.

\section{Mode of delivery}

The rate of cesarean section in general population is 10 $15 \%$. Literature suggests that there is an increase in the rate of cesarean section in patients with PROM as compared to normal pregnancies without any complication. This usually occurs due to increased chances of fetal distress, oligohydramnios and abruption. ${ }^{4,5}$ Similar results are shown in the present study with $22 \%$ patients in confirmed leaking (study Group 1) and $10 \%$ patients in the unconfirmed leaking (study Group 2) having cesarean section. The difference between the groups was not statistically significant ( $p$ value 0.102 ). 


\section{Apgar score and NICU admission}

Various studies show that babies of mothers who had PROM, with or without associated oligohydramnios have increase in the rate of NICU admission, low Apgar scores and increased neonatal deaths. This is mainly attributed to prematurity, neonatal sepsis, increased chances of hyaline membrane disease and fetal distress.

Apgar score reflects the infant's respiratory, circulatory and neurological status. The 5-minute Apgar score is more useful than 1-minute score in reflecting the infant's condition. ${ }^{3-5}$ The 5-minute Apgar score was $\geq 7$ in $74 \%$ and $86 \%$ patients in the confirmed leaking group and unconfirmed leaking group in the present study. The difference between the groups is not statistically significant ( $p$ value 0.134). This reflects that the respiratory complications like fetal distress and hyaline membrane disease were low which could be because of the mean gestational age of more than 34 weeks in both the groups. Results in the present study show 36\% and $30 \%$ NICU admission in patients with confirmed leaking (study Group 1) and unconfirmed leaking (study Group 2) respectively. The difference between both the group was not statistically significant.

In the present study the mean gestational ages in the study Group 1 and 2 were 34.8 and 34.9 respectively. The higher rates of NICU admission was mainly attributed to prematurity and very few (8\% and $2 \%$ ) patients had oligohydramnios which could affect the NICU admission rates.

\section{CONCLUSION}

Estimation of creatinine in vaginal wash fluid was found to have a specificity of $100 \%$ but sensitivity of $76 \%$. The vaginal wash fluid urea levels of $>8.55 \mathrm{mg} / \mathrm{dl}$ detected PROM with a sensitivity and specificity of $100 \%$. The negative and positive predictive value for the presence of PROM were also $100 \%$. The predictive accuracy of vaginal wash fluid urea and creatinine did not decrease with increase in duration since leaking. Further gestational age specific studies are needed to confirm vaginal wash fluid creatinine as a marker of PROM meanwhile it can be used as a supportive test. Though the present study could not find any correlation of vaginal, wash fluid urea and creatinine with gestational age further studies could be done on a larger sample size to confirm this.

Funding: No funding sources Conflict of interest: None declared

Ethical approval: The study was approved by the Institutional Ethics Committee

\section{REFERENCES}

1. ACOG Committee on Practice Bulletins-Obstetrics, authors. Clinical management guidelines for obstetrician-gynecologists. (ACOG Practice Bulletin No. 80: premature rupture of membranes). Obstet Gynecol. 2007;109:1007-1019.

2. Gallot D, Guibourdenche J, Sapin V, Goffinet F, Doret M, Langer B, et al. Which biological test to confirm rupture of membranes? J Gynecol Obstet Biol Reprod. 2012;41:115-21.

3. Méhats C, Schmitz T, Marcellin L, Breuiller-Fouché M. Biochemistry of fetal membranes rupture. Gynecol Obstet Fertil. 2011;39:365-9.

4. Martinuzzi K, Mariona F. Comment and reply on: The clinical significance of a positive AmniSure test in women with term labor with intact membranes. J Matern Fetal Neonatal Med. 2011;24:654.

5. Erdemoglu E, Mungan T. Significance of detecting insulin-like growth factor binding protein-1 in cervicovaginal secretions: comparison with nitrazine test and amniotic fluid volume assessment. Acta Obstet Gynecol Scand. 2004;83:622-6.

6. Caughey AB, Robinson JN, Norwitz ER. Contemporary diagnosis and management of preterm premature rupture of membranes. Rev Obstet Gynecol. 2008;1(1):11-22.

7. Kafali H, Oksuzler C. Vaginal fluid urea and creatinine in diagnosis of premature rupture of membranes. Arch Gynecol Obstet. 2007;275:157-60.

8. Gurbuz A, Karateke A, Kabaca C. Vaginal fluid creatinine in premature rupture of membranes. Int $\mathbf{J}$ Gynecol Obstet. 2004;85(3):270-1.

9. Robson MS, Turner MJ, Stronge JM, O’Herlihy CO. Is amniotic fluid quantitation of value in the diagnosis and conservative management of prelabour membrane rupture at term? Br J Obstet Gynaecol. 1990;97:324-8.

10. Oliveira FR, Barros EG, Magalhães JA. Biochemical profile of amniotic fluid for the assessment of fetal and renal development. Braz J Med Biol Res. 2002;35(2):215-2.

11. Deshpande TV, Harding PG, Jaco NT. Estimation of gestational age from study of amniotic fluid and clinical assessment. Can Med Assoc J. 1977;117(8):886-90.

Cite this article as: Sharma A, Sharma R, Agarwal T. Evaluation of urea and creatinine levels in vaginal wash fluid for the diagnosis of premature rupture of membranes. Int J Reprod Contracept Obstet Gynecol 2020;9:3449-57. 